\title{
El modelo de desarrollo económico de la India
}

DOI: $10.32870 /$ mycp.v4i14.646

Vikram Chadha***

\ ntroducción

Al momento de su independencia en 1947 , la economía india se encontraba en desorden debido a la desgastante explotación que efectuaron los imperialistas británicos. De 1860 a 1945, la economía no creció a más de $0.5 \%$ anual. El índice del ingreso per cápita sólo creció de 100 en 1913 a 109 rupias en 1946. La producción agrícola se incremento sólo en $10 \%$ de 1893 a 1946, por lo que el índice de producción de granos experimentó un decremento de 100 a 93 toneladas y la disponibilidad per cápita cayó en $32 \%$, lo que provocó una pobreza extrema de las masas (Chandra, 1992). Igualmente, el sector industrial presentaba los rasgos característicos de atraso, un consumo insignificante de bienes de base industrial y la ausencia virtual de bienes de capital y manufacturas de industria pesada.

Debido a la pobreza y a una tasa de ahorro interno inferior, el promedio de capital de formación era alarmantemente bajo. Salvo por las vías férreas, la infraestructura industrial prácticamente no existía y la educación y capacitación técnica habían sido pasadas por alto deliberadamente por los gobernantes británicos.

Con semejante retraso en la economía india al momento de su independencia, se tenía la tarea de consolidar y reconstruir a una economía sitiada, para prepararla y enfocarla hacia un desarrollo económico rápido y la modernización. El camino era difícil y tenía que abordarse con precaución, teniendo en cuenta el largo periodo histórico de explotación que se experimentó en las manos de un avaro sistema capitalista mundial.

* Investigador visitante de la Univesidad Deguru Nanak Dev, Amiritsar,India.

** Traducción de Osvaldo Monreal
Con influencias del modelo ruso de planificación, desde 1951, la India dio inicio a sus planes quinquenales. Estos Planes tenían que incorporar una determinada estrategia de desarrollo. Los dirigentes de la economía por lo menos evitaron la ambigüedad en cuanto a que el plan de estrategia debía esforzarse por generar confianza y crecimiento interior y asi evitar la independencia innecesaria de las economías desarrolladas de Occidente. En la economía india se procedió con cautela debido a las consecuencias destructivas del sistema capitalista mundial.

Por ello la estrategia de desarrollo debía procurar esencialmente independencia y confianza económica.

\section{El patrón de asignación sectorial en los planes}

El Primer Plan Quinquenal (1951-1956) era un plan modesto y esencialmente aspiraba a reconstruir la economía, a causa del daño provocado por la guerra, el hambre de 1943 y la división del país. El Plan asignó $19.6 \mathrm{mil}$ millones de rupias para el gasto público y 18 mil millones de rupias para el gasto de reconstrucción del sector privado (gobierno de la India, 1996-1997). El gasto en el sector público estaba principalmente dirigido a crear infraestructura social para el desarrollo y comprendía proyectos de irrigación, suministro de energía y transporte. Otras actividades directamente productivas, sobre todo para producir bienes de consumo, se dejaron en manos del sector privado.

Así el desarrollo agrícola obtuvo $37 \%$ de las erogaciones del presupuesto, la industria pesada sólo $5 \%$, transporte $26.5 \%$ y para la generación de energía se asignó $7.5 \%$. La idea de crear confianza en la economía interna era evidente, dado que sólo $10 \%$ de los recursos 
para el presupuesto del plan se obtuvieron de fuentes externas.

El Segundo Plan (1956-1961) tenía como objetivo un crecimiento a largo plazo; y era un plan muy ambicioso. Alentado por el éxito del primer plan, el desembolso para éste aumentó en $90 \%$, es decir 46.7 billones de rupias para el sector público y 31 mil millones de rupias para el privado (gobierno de la India, varias publicaciones). En este Plan, se dió mayor énfasis a la industria, sobre todo a las intensivas en capital. Se establecieron varias industrias de gran magnitud en sectores claves, incluyendo a los del acero, cemento, fertilizantes, refinerías de aceite, maquinas y herramientas.

Así, considerando que el gasto del sector público en la agricultura era justamente un 20 $\%$ del gasto total, la asignación para la industria era de $24 \%$, transporte y comunicación $27 \%$ y para el sector energía $10 \%$. Otro rasgo del Plan era el fortalecimiento del sector público, particularmente la industria pesada y el sector de energía. Para financiar la inversión orientada a la industria pesada se recurrió a fuentes externas, que proveyeron $22.5 \%$ de las finanzas requeridas. No obstante, el plan no obtuvo los resultados deseados; el crecimiento de la economía disminuyó, la pobreza aumentó, la producción de bienes de consumo experimentó un déficit, la balanza de pagos era adversa, pero aumentaron las tasas de formación de capital de manera importante, lo cual dio a la ndustria una base durable y fortaleció a la economía.

La estrategia de desarrollo del Segundo plan quinquenal tuvo continuidad en el tercer plan (1961-1966). En este, se asignaron 86.3 mil millones de rupias para el gasto del sector público y 41 mil millones de rupias para el privado. En total las erogaciones por concepto de presupuesto aumentaron $66.5 \%$ más que el segundo plan. Se continuó haciendo énfasis en el desarrollo de la industria pesada dándosele $23 \%$ a diferencia del $20.5 \%$ que se destinó para la agricultura.

El tercer plan estaba más enfocado al sector energía, razón por la que se le asignó un $4.5 \%$ más que en el segundo plan. Sin embargo, para financiar la inversión que se requería para industria pesada y tecnología sofisticada, se hizo necesario acudir al endeudamiento externo. Así $28 \%$ del gasto se reunió de fuentes externas. Tampoco este plan logró sus objetivos: sacó a flote una agricultura en retraso que difícilmente podía sostener el progreso de la economía. El fracaso de la agricultura era el factor más importante el retroceso general del período 19651967 . La disminución en la proporción de crecimiento después de 1965 tuvo como resultado un exceso de capacidad en la industria. Muchos establecimientos comerciales dejaron de operar para suprimir la presión de los costos anormales. De esta manera, la India atravesó una curiosa recesión que combinaba características de inflación y depresión.

Debido al fiasco del Tercer Plan, los economistas se desilusionaron de los cinco años que habían planeado, y durante el periodo de 1966 a 1969 el país tuvo tres planes anuales. Durante estos planes anuales, el desembolso del sector público sumó 66.3 mil millones de rupias. El énfasis en la agricultura y sectores relacionados aumentó durante estos tres planes; $24 \%$ del presupuesto estaba destinado para este sector. El desembolso industrial también aumentó a $25 \%$ y el sector de generación de energía todavía obtuvo una porción mayor, con $18 \%$ del total. Pero la asignación para transporte disminuyo drásticamente a $18.5 \%$ y los sectores sociales obtuvieron solamente $14.5 \%$ del desembolso total. Debido a un déficit en generación de recursos para el Tercer Plan, las fuentes externas de fondo tenían que cubrir $36 \%$ del desembolso. A pesar de los mejores esfuerzos de la India, no podría mantenerse una independencia de las fuentes externas de financiamiento.

Teniendo en cuenta el fracaso de los planes anteriores, el Cuarto Plan Quinquenal elaborado para el periodo 1969-1974 disminuyó las proyecciones a largo plazo sustancialmente y puso mayor énfasis en lograr confianza interna. Sin embargo hubo un $112 \%$ de aumento en el presupuesto en comparación con el que se destinó para el Tercer Plan, y se asignaron $161.6 \mathrm{mil}$ millones de rupias para el 
desembolso del sector público, mientras que en el privado se gastaron 89.8 mil millones de rupias. El presupuesto para el desarrollo industrial se redujo considerablemente a $19.7 \%$ del desembolso del Plan, mientras que el del sector de la agrícola se mantuvo en un $23.5 \%$. De igual manera, el presupuesto destinado para energía, transporte y c o m u n i c a c i o n e s, respectivamente, se mantuvo en $18.5 \%$ y $19.5 \%$. El gasto en los servicios sociales, incluyendo la educación, salud y desarrollo rural, se reforzó considerablemente con $19 \%$ del desembolso total. El fondo externa del plan se disminuyó drásticamente a sólo $13 \%$, con el fin de concretar el objetivo de confianza en la economía interna.

El Quinto Plan Quinquenal (1974-1979) tuvo problemas desde su concepción, cuando los mayores cambios en el ambiente económico se hicieron visibles. Muchos de los programas de plan tuvieron que ser archivados para establecer un " Nuevo Programa Económico “, popularmente conocido como el Programa de veinte puntos, anunciado en 1975. En desembolso financiero aumentó en $174.5 \%$ de lo que se había destinado para el Cuarto Plan. Se asignaron 423 mil millones de rupias para el sector público y 270.5 mil millones de rupias para el privado fuera del desembolso total. La importancia de la agricultura continuó con una asignación del $22 \%$ de fondos. Pero el gasto planeado para el sector industrial se incremento a $24.5 \%$. La energía, transporte y servicios sociales continuaron teniendo casi los mismo recursos que en planes anteriores. Se redujo el financiamiento del Plan por medio de fuentes externas al mínimo de $7.5 \%$ lo cual fue un fuerte apoyo para la meta de la economía interna. Sin embargo, debido a que el gobierno del centro a fue derribado, el Quinto Plan se acabó un año antes del periodo de terminación.
Los objetivos mayores del Sexto Plan Quinquenal (1980-1985) eran lograr una media proporción de crecimiento anual de $5.2 \%$ y fortalecer el impulso de modernización para lograr una economía sólida al interior y confianza en la tecnología interna, así como la reducción progresiva en la incidencia de la pobreza y el desempleo. El Plan asignó1104 mil millones de rupias para el desembolso del sector público y $777 \mathrm{mil}$ millones de rupias para el privado. Para reducir dependencia externa, sólo 7.7 $\%$ del gasto del Plan serían reunidos de las fuentes extranjeras. En el Sexto Plan, el presupuesto marcado aumentó $150 \%$, del asignado para el Plan anterior, mientras el desembolso industrial fue de $26.5 \%$ del total. La asignación de recursos para la energía, transporte y comunicación y otro sectores de servicios social se redujo marginalmente. Los objetivos del Plan casi pudieron lograrse de no ser por pérdidas en las cosechas y un progreso industrial insatisfactorio.

El Séptimo Plan Quinquenal a (19851990) estaba diseñado para un crecimiento medio anual de $5 \%$, con una larga perspectiva hasta el año 2000. las asignaciones del presupuesto para el sector público sumaron 2229,2 mil millones de rupias, mientras que 1681 mil millones se destinaron a gastos en el sector privado. Todo el gasto planeado era 102 $\%$ más alto que en el Plan anterior y $8.5 \%$ del desembolso estaba programado para cubrirse por fuentes externas. El gasto planeado en agricultura e industria se redujo marginalmente con $20 \%$ y $23.5 \%$ del desembolso total respectivamente para estos sectores, pero se reforzó el gasto en servicios sociales significativamente a $21.5 \%$ del total. Al final de este Plan el objetivo de crecimiento anual de $5 \%$ se excedió y se dio una mejora perceptible en ingresos agrícolas, en el consumo per cápita, y un declive en la proporción capital-producción que era de comprenderse. Pero desde que el 
gasto del Plan estaba financiado con déficit, resultó con precios más altos, problemas con la balanza de pago y crisis fiscal.

El Octavo Plan Quinquenal a (1992-1997) perseguía una estrategia de crecimiento basada en el desarrollo de la infraestructura, disminución de la pobreza, generación de empleo, control de la población y salud para todos. Esta vez se asignaron 4341 mil millones de rupias como desembolso para el sector público y 4370 mil millones de rupias para el privado. Era evidente, por el patrón que seguía el presupuesto, que desde que la economía se estaba liberalizando mediante la supresión de controles en el sector privado y abreviando la importancia del sector público, el desembolso de aquél excedió en este. En todos se refleja un 95 $\%$ en el desembolso del Plan. Se aumentaron los gastos en servicios sociales notablemente a 23 $\%$ del presupuesto total.

El Noveno Plan Quinquenal, que es el actual (1997-2002), está diseñado para dar confianza al desarrollo de la infraestructura, disminuir la pobreza, promover la agricultura y generar empleo. En línea con la liberalización y el proceso de la reforma hacia la privatización, se asignaron 13100 mil millones de rupias mientras el desembolso del sector público es de 8750 mil millones de rupias. Se han minimizado las fuentes externas de fondos a $7 \%$ del total del presupuesto para el plan y la financiación deficitaria esta programada para llegar a un nivel cero. La asignación para impulsar el sector energía se ha aumentado a $8 \%$ del total, el gasto en transporte sea reprogramado al igual que los servicios sociales, respectivamente en $20 \%$ y $27 \%$ del total.

\section{La estrategia de desarrollo en los planes}

Una estrategia de desarrollo incorpora un sistema de extremos que pueden seguirse con una técnica definida. La estrategia implica una opción deliberada esencialmente - la eleción del rubro, su plazo y la manera en que se va a atacar el problema (Patel, 1992) y consiste en tres componentes: la movilización del recurso, las prioridades para la asignación de los recursos movilizados, y una política sólida para asegurar un despliegue eficaz de recursos en los sectores de prioridad.

Aunque el Primer Plan no tenía un término de perspectiva a largo plazo, aun así su objetivo era aumentar el desarrollo potencial de la agricultura en la primera fase, para tener una base fuerte para una industrialización rápida en las fases subsecuentes.

El Segundo Plan sentó las bases de estrategia de desarrollo en el sentido real. Encontraba su base principalmente en la estadística y la economia ( P.C. Mahalanobis). Este modelo se volvió la guía para todos los planes subsecuentes. La Segunda estrategia del Plan era construir una economía confiable al interior, por lo que notablemente se trataba de evitar una estrategia del laissez-faire para la industrialización india, como también a descentralizar un pueblo orientado por una estrategia de desarrollo agrícola. Así los rasgos más importantes del modelo indio de desarrollo económico pueden resumirse en los siguientes puntos.

\section{a) Economía Mixta}

aunque la india emuló el modelo ruso de desarrollo planeado, siendo una democracia parlamentaria no podría permitirse el lujo de eclipsar la iniciativa privada. Así, la estrategia de desarrollo industrial, a pesar de dar más importancia al sector público , nunca minó el papel del privado. La " resolución en de la política industrial “ de 1956 demarcó las áreas de operación del sector público y del sector privado claramente. Las industrias importantes y básicas, la infraestructura y utilidades públicas y el desarrollo de industrias estratégicas, estarían completamente dentro de la esfera del sector público, mientras que los bienes de consumo y servicios irían de la mano. Así, aunque el sector público tendría un papel dominante en el proceso de desarrollo industrial, todavía no suplantaría el papel del sector privado. En otras palabras, el modelo del Plan adoptó un acercamiento económico mixto a desarrollo. 


\section{b) Confianza Interior}

La estrategia de desarrollo que los analistas indios discutían era la independencia, esencialmente cautos por el daño infligido en la India por los imperialistas británicos y sus políticas económicas avariciosas, que habían ahuecado y estrellado la economía india. Para los estadistas y políticos que querían que la India creciera independiente de la dinámica del desarrollo económico mundial, se logró la meta de confianza interior, que fue vista como la conclusión de un objetivo y una herramienta estratégica para proteger la industria doméstica de la competencia extranjera mediante aranceles y medidas no-arancelarias. Así, la estrategia de lograr la auto confianza se reflejó en todas las políticas económicas como la política de importación-exportación, política de desarrollo de tecnología, política de la autorización industrial y régimen de política monetaria y fiscal.

\section{c) Papel complementario de las industrias grandes y pequeñas}

Aunque el Segundo Plan dio énfasis en el desarrollo de capital intensivo, las industrias básicas y pesadas aún tenían rol incipiente, generando sueldo bajos. De hecho, las industrias pequeñas serían protegidas de la competencia de industrias domésticas y extranjeras manteniendo los mercados protegidos, en y un sistema de incentivos monetarios y fiscales. Se pensó que en el proceso de desarrollo de bienes de capital e industrias de ingeniería pesada, la generación de empleo y la producción de bienes de consumo para la masa se dirigirían a la esfera de las industrias pequeñas. Además, se desarrollarían en una relación auxiliar con el sector industrial y ayudarían a que se diera una distribución justa de recursos.

\section{d) Digresiones en las estrategias de los planes de desarrollo}

Aparte de los pilares anteriores de modelo de desarrollo de la India, ha habido, de vez en cuando, ciertos cambios en la estrategia de desarrollo a seguir, para hacer frente a las compulsiones de las variadas circunstancias económicas

e) La incorporación del modelo de la demanda en la estrategia de desarrollo

Durante el Cuarto Plan se comprendió que un aumento en el crecimiento del PIB y una proporción alta de acumulación de capital no podría mantener una condición suficiente para mejorar la calidad de vida y el consumo que necesitan las masas. Así, durante este Plan, se dio una mezcla de inversión escogida con base en el modelo de demanda para el consumo final; el Cuarto Plan estaba basado en un modelo de consistencia de intersectorial una variante del modelo de insumo producción de Leontief.

\section{f) Incorporación de redistribución con estrategia de crecimiento}

Mientras se formulaba el Segundo Plan, los estadistas presumían que la inversión pesada en el sector industrial llevaría a un crecimiento perceptible de ingreso nacional, que en el periodo subsecuente automáticamente se distribuiría a las masas y levantaría sus ingresos y niveles de consumo. Pero después se encontró que el beneficio de crecimiento no había llegado hasta las masas, debido a las estructuras institucionales que inhibieron este proceso. Así, durante el Quinto Plan, como una medida correctiva, la estrategia de desarrollo india previó el consumo necesario para las personas de escasos recursos y comenzaron varios programas para aumentar el poder adquisitivo adicional entre los pobres; se proveyó un mayor sueldo a los pobres. De acuerdo con las variables económicas y no-económicas, se identificó a la pobreza y se la incorporó en el modelo de desarrollo para construir una estrategia de redistribución explícita . Se contempló algunas variables, como los requisitos de las personas, la participación de las masas en el desarrollo y la reducción de la desigualdad del ingreso entre las clases; de alguna manera se trataba de la incluir todos los sectores de la población, aunque se daba prioridad a los más afectados. 
Cuadro 1

Presupuestos financieros de los planes en la India

(miles de millones de rupias)

\begin{tabular}{|c|c|c|c|c|}
\hline Plan & Periodo & $\begin{array}{c}\text { Gastos del } \\
\text { sector } \\
\text { público }\end{array}$ & $\begin{array}{l}\text { Gasto del } \\
\text { sector } \\
\text { privado }\end{array}$ & $\begin{array}{c}\% \text { de } \\
\text { incremento } \\
\text { en el total }\end{array}$ \\
\hline Primero & $1951-56$ & 20 & 18 & $\begin{array}{lll}- & - \\
-1\end{array}$ \\
\hline Segundo & $1956-61$ & 47 & 31 & 90 \\
\hline Tercero & $1961-66$ & 86 & 41 & 67 \\
\hline Planes anuales & $1966-69$ & 66 & & - \\
\hline Cuarto & $1969-74$ & 162 & 90 & 112 \\
\hline Quinto & 1974-79 & 423 & 271 & 148 \\
\hline Sexto & $1980-85$ & 1,105 & 777 & 150 \\
\hline Séptimo & $1985-90$ & 2,229 & 1,682 & 102 \\
\hline Planes anuales & $1990-92$ & 1,424 & . & - \\
\hline Octavo & $1992-97$ & 4,341 & 4,370 & 95 \\
\hline Noveno & 1997-02 & 8,750 & 13,100 & 100 \\
\hline
\end{tabular}

Fuente: Government of India ( Various Issues ),

Basic Statistics Relating to the Indian Economy,

New Delhi : CSO.

Cuadro 2

Fuentes de financiamiento de los planes en la India

(porcentaje)

\begin{tabular}{lcccccccccc}
\hline \multicolumn{1}{c}{ Fuente } & 1er Plan & $2^{\circ}$ Plan & 3er Plan & Planes anuales & $4^{\circ}$ Plan & $5^{\circ}$ Plan & $6^{\circ}$ Plan & $7^{\circ}$ Plan & $8^{\circ}$ Plan & $9^{\circ}$ Plan \\
\hline $\begin{array}{l}\text { Fuentes } \\
\text { internas }\end{array}$ & 73.4 & 57.1 & 58.5 & 54 & 74.4 & 78.1 & 78.1 & 75.8 & 87.18 & 93.1 \\
$\begin{array}{l}\text { Fuentes } \\
\text { externas }\end{array}$ & 9.6 & 22.5 & 28.2 & 35.9 & 12.9 & 7.5 & 7.7 & 8.4 & 8.2 & 6.9 \\
$\begin{array}{l}\text { Déficit } \\
\text { financiero }\end{array}$ & 17 & 20.4 & 13.3 & 10.1 & 12.7 & 14.4 & 14.2 & 15.8 & 4.62 & 0 \\
Total & 100 & 100 & 100 & 100 & 100 & 100 & 100 & 100 & 100 & 100 \\
\hline
\end{tabular}

Fuente: Government of India ( Various Issues ),

Basic Statistics Relating to the Indian Economy,

New Delhi : CSO.

Cuadro 3

Patrón de localización de recursos en los planes (porcentaje)

\begin{tabular}{|c|c|c|c|c|c|c|c|c|c|c|}
\hline $\begin{array}{l}\text { Agricultura y } \\
\text { sectores } \\
\text { relacionados: } \\
\text { irrigación y control }\end{array}$ & & & & & & & & & & \\
\hline de aqua & 37 & 20.9 & 20.5 & 23.8 & 23.3 & 22.1 & 22.1 & 20.2 & 20.6 & 19.4 \\
\hline Electricidad & 7.6 & 9.7 & 14.6 & 18.3 & 18.6 & 18.8 & 16.7 & 17.4 & 18.7 & 25.4 \\
\hline $\begin{array}{l}\text { Industria } \\
\text { Transportes y }\end{array}$ & 4.9 & 24.1 & 22.9 & 24.7 & 19.7 & 24.3 & 26.5 & 23.7 & 18.8 & 8.2 \\
\hline Comunicaciones & 26.4 & 27 & 24.6 & 18.5 & 19.5 & 17.4 & 16 & 17.1 & 18.7 & 19.8 \\
\hline Sevicios sociales & 24.1 & 18.3 & 17.4 & 14.7 & 18.9 & 17.4 & 18.6 & 21.6 & 23.2 & 27.2 \\
\hline Total & 100 & 100 & 100 & 100 & 100 & 100 & 100 & 100 & 100 & 100 \\
\hline
\end{tabular}

Fuente: : Government of India ( Various Issues ), Economic Survey, New Delhi: Ministry of Finance

Government of India ( 1997 ) Annual Report 1996-97, New Delhi : Planning Commission

Government of India ( 1997 ), Draft Ninth Five Year Plan 1997-2002, New Delhi: Planning Commission 


\section{g) La nueva política económica y eficacia}

Desde julio de 1991, la India introdujo su Nueva Política Económica (NEP), la cual comprende varias medidas y cambios para mejorar el sistema, sobre todo abreviando el costo de gobernación y administración; se esfuerza por distribuir, mediante el mecanismo regulador que involucra una multitud de competidores y que impide controles, y crear un ambiente más competitivo para los negocios. Esto se logrará quitando las barreras del mercado y las restricciones en el crecimiento de las empresas. La Nueva Política de comercio busca mejorar competitividad internacional, eliminando o diluyendo el depósito de arancel y regulaciones no arancelarias.

El sector privado está dándose un espacio más grande para operar, excepto en las áreas reservadas para el sector público, y el último tendrá que competir con el anterior en base a la eficacia y productividad. El sistema de la autorización industrial ha cobrado importancia y ha dado énfasis al liberar el sector público (PSUs).

No obstante, el nuevo ambiente de la política no deja a un lado completamente el papel del Estado. De hecho, el gobierno tendrá la responsabilidad primaria del desarrollo de infraestructura física, como irrigación, energía, transporte, comunicación e infraestructura social, alfabetización, salud, control de la población, ambiente y programas para cuidar de los pobres y las secciones perjudicadas. De esta manera la planeación se dirigirá al mercado, en lugar de al control estatal, con el objetivo de lograr crecimiento y eficacia, mientras se mantiene la estabilidad macroeconómica.

\section{Fuentes}

Chandra, B. , “ The colonial legacy “, in B. Jalan (ed. ), The indian economy, New Delhi, 1992 p. 12.

Desai, B. M. and N.V. Namboodiri , " policy strategy and instruments for alleviating Rural poverty “, Economic and political weekly, vol. XXXIII, no. 41, Oct. 16., 1998

Government of India, Second Five Year Plan, New Delhi, 1956, p. 259.

Government of India (1997 ), Economic survey, New Delhi Government of India ( 1997 ), Draft Ninth Five Year Plan, New Delhi.

Government of India ( 1997 ), Annual report, New Delhi. Government of India ( various issues ), Basic statistics relating to the indian economy, New Delhi.

Patel, I.G. ( 1992 )," The strategy of indian planning “, in P. Chaudhuri (ed. ), Aspects of indian economic development , New Delhi , p.116. 